\title{
Total astragalosides decrease apoptosis and pyroptosis by inhibiting enterovirus 71 replication in gastric epithelial cells
}

\author{
XIAOYAN ZHANG ${ }^{1 *}$, JINFANG HAO $^{1 *}$, CHENXI SUN $^{1}$, JIANPING DU $^{1}$, QIAN HAN $^{1}$ and QINGSHAN LI ${ }^{1,2}$ \\ ${ }^{1}$ Department of Laboratory Medicine of Fenyang College, School of Pharmaceutical Science, Shanxi Medical University, \\ Taiyuan, Shanxi 030001; ${ }^{2}$ Shanxi Key Laboratory of Innovative Drugs for The Treatment of Serious Diseases Based on \\ Chronic Inflammation, Shanxi University of Chinese Medicine, Jinzhong, Shanxi 030619, P.R. China
}

Received April 19, 2021; Accepted October 11, 2021

DOI: $10.3892 /$ etm.2022.11162

\begin{abstract}
Enterovirus 71 (EV71) is one of the primary pathogens involved in severe hand, foot and mouth disease in children. EV71 infection causes various types of programmed cell death. However, there are currently no clinically approved specific antiviral drugs for control of EV71 infection. Astragalus membranaceus (AM), a Traditional Chinese medicine, has been used in antiviral therapy in China. The aim of the present study was to determine whether total astragalosides (ASTs), bioactive components of AM, protect against EV. DAPI nuclear staining was used to observe morphological changes of the nucleus and the protective effect of ASTs, which revealed that the nucleus shrank following EV71 infection, while ASTs reversed it. Cell Counting Kit-8 assay found that human normal gastric epithelial cell (GES-1 cell) viability decreased following EV71 infection, while lactate dehydrogenase (LDH) assay showed that EV71 infection induced GES-1 cell damage. Western blotting was used to measure the expression levels of apoptosis and pyroptosis marker protein to determine whether EV71 infection induced apoptosis and pyroptosis in GES-1 cells. Reverse transcription-quantitative PCR was used to determine the anti-EV71 effect of ASTs. The results showed that ASTs protected GES-1 cells from EV71-induced cell apoptosis and pyroptosis. Furthermore, the present data demonstrated that the protective effect of ASTs was exerted by suppressing EV71 replication and release. These findings suggested that ASTs may represent a potential antiviral agent for the treatment of EV71 infection.
\end{abstract}

Correspondence to: Professor Xiaoyan Zhang or Professor Qingshan Li, Department of Laboratory Medicine of Fenyang College, School of Pharmaceutical Science, Shanxi Medical University, 56 Xinjian South Road, Taiyuan, Shanxi 030001, P.R. China

E-mail: 931326464@qq.com

E-mail: sxlqs2012@163.com

*Contributed equally

Key words: total astragaloside, enterovirus 71, apoptosis, pyroptosis, replication

\section{Introduction}

Hand, foot and mouth disease (HFMD) broke out in Fuyang, Anhui in 2008, resulting in many children's deaths and since then, the prevalence of HFMD has been rising rapidly in China (1). HFMD primarily infects children under the age of 6 years and is caused by Coxsackie virus A16 (Cox A16) and enterovirus 71 (EV71) (2). The rapid progression of HFMD may lead to serious nervous system complications involving cerebritis, meningitis and high mortality $(3,4)$. These severe cases are primarily caused by EV71 infection. However, the pathogenic mechanism of EV71 infection is not yet completely understood and there is no specific drug against EV71 infection.

Viral infection induces several types of programmed cell death (PCD), such as apoptosis (5) and pyroptosis (6). Certain types of PCD are part of the stress response used by the body to eliminate the virus; however, other types promote viral infection and spread in the body. For example, hepatitis B virus induces apoptosis of liver cancer cells (7) and EV71 infection induces pyroptosis (8). In the present study, it was hypothesized that the pathogenesis of EV71 infection may be associated with apoptosis and pyroptosis of infected cells.

Apoptosis is an autonomous form of PCD that is controlled by genes, such as Bcl-2, p53, c-myc and caspases $(9,10)$. The morphological manifestations of apoptosis include decreased cell volume, rupture of the nuclear membrane and nucleolus, intact cell membrane structure and separation of apoptotic bodies from apoptotic cells (11). Apoptosis does not lead to inflammation in the surrounding cells (12). However, inhibiting EV71-mediated apoptosis can protect cells (13).

Pyroptosis is a newly identified type of PCD that was discovered by Brennan and Cookson in salmonella-infected macrophages (14). Pyroptosis is identified by the presence of swollen cells, which rupture and cause the release of IL-1 $\beta$ and IL-18 (15), thereby inducing an inflammatory response in the body (16). The inflammatory response triggered by EV71 is the primary cause of high levels of inflammation observed in severe HFMD $(17,18)$. Considering the association between pyroptosis and inflammation, it was hypothesized that suppressing EV71-mediated pyroptosis may lead to identification of novel anti-inflammatory agents for the treatment of HFMD. 
Current anti-inflammatory therapies include steroidal and non-steroidal treatments, both of which have side effects $(19,20)$. The aim of the present study was to identify anti-inflammatory ingredients in natural plants, such as those used in Traditional Chinese medicine. Throughout years of clinical practice, the effect of Astragalus membranaceus (AM) on inflammation has gradually been recognized (21). Total astragalosides (ASTs), the primary active ingredients of AM, are used in the treatment of nephrotic syndrome (22) and can inhibit the proliferation of gastric cancer cells (23), prevent oxidative damage (24) and protect against inflammation (25). Therefore, the present study examined whether ASTs attenuate EV71-induced cell injury by investigating the effect of ASTs on cell viability, viral replication and release and cell apoptosis and pyroptosis. The present study aimed to enhance understanding of the pathogenic mechanism of EV71 and develop novel strategies for EV71 infection.

\section{Materials and methods}

Cell lines and culture. Human normal gastric epithelial cell (GES-1 cells) and human rhabdomyosarcoma cells (RD) were obtained from Fenyang College of Shanxi Medical University (Fenyang, China) and cultured in DMEM (Boster Biological Technology) supplemented with 10\% FBS (Biological Industries Technology), $1 \%$ penicillin and $1 \%$ streptomycin (Boster Biological Technology). The cells were maintained in an incubator (Thermo Fisher Scientific, Inc.) at $37^{\circ} \mathrm{C}$ with $5 \% \mathrm{CO}_{2}$.

Tissue culture infectious dose 50 (TCID50) assay. EV71 was generously supplied by Professor Zhendong Zhao from the Chinese Academy of Medical Sciences \& Peking Union Medical College, Institute of Pathogen Biology (Beijing, China). The EV71 titer was measured using TCID50 assay. Steps are as follows: RD cells were seeded into 96-well plates (1x10\% /well) and incubated overnight at $37^{\circ} \mathrm{C}$ in an incubator. EV71 was serially diluted $1 \times 10^{1}-1 \times 10^{11}$ times in centrifuge tubes and then added to the 96 -well plates ( 8 repeated/dilution). The 96-well plates were observed under the light inverted microscope (magnification, $\mathrm{x} 40$ ) once every day for 5 days and $\sim 50 \%$ of the cytopathic effect in the wells was recorded. TCID50 results were calculated according to the Reed-Münch method (26) using the following formula: Plaque-forming units $=0.7 \times$ TCID50.

Viral infection. GES-1 cells were seeded into 6-well plates (1x10\% /well) and infected with EV71 at a specified multiplicity of infection (MOI; 0, 1, 3 and 5) at $37^{\circ} \mathrm{C}$ for $24 \mathrm{~h}$, and infected cells and supernatant were collected at 2,000 $\mathrm{x} \mathrm{g}$ for $3 \mathrm{~min}$ at room temperature. Proteins were extracted from cells and analyzed using western blotting. The aforementioned supernatant was collected to measure the virus titer.

Antibodies and reagents. BCA protein detection kit (cat. no. AR0146) and ECL western blot detection kit (cat. no. AR1171) were obtained from Boster Biological Technology. Total astragalosides (cat. no. SA8600) were purchased from Beijing Solarbio Science \& Technology Co, Ltd. Horseradish peroxidase-conjugated goat anti-rabbit
IgG (H+L) antibody (cat. no. CW0103) was purchased from CoWin Biosciences. Anti-caspase-3 (cat. no. WL02117), anti-NLR family, pyrin domain containing 3 (NLRP3; cat. no. WL02635), anti-cleaved (c)-caspase-3 (cat. no. WL01992) and anti-pro-IL-1 $\beta$ (cat. no. WL02257) antibodies were purchased from Wanleibio Co., Ltd. Anti-gasdermin D protein (GSDMD; cat. no. A17308) and anti-pro-caspase-1 (cat. no. A0964) antibodies were obtained from ABclonal Biotech Co., Ltd. Anti-PARP (cat. no. AP102), anti-c-PARP (cat. no. AF1567) antibodies, Lactate dehydrogenase (LDH) detection kit (cat. no. C0017), DAPI (cat. no. C1002) and BeyoRT II First Strand cDNA Synthesis kit (cat. no. D7168) were obtained from Beyotime Institute of Biotechnology. Anti-c-caspase-1 (cat. no. BS65650) antibody was obtained from Bioworld Technology,Inc. Anti-VP1 (cat. no. GTX132313) antibody was purchased from GeneTex, Inc. Cell Counting Kit-8 (CCK-8; cat. no. 40203ES60) assay and Hieff Universal Blue qPCR SYBR Green Master mix (cat. no. 11141ES) were purchased from Yeasen Biotech Co., Ltd.

Cell treatment and morphological observation. GES-1 cells were plated into 6 -well plates $\left(1 \times 10^{6} /\right.$ well) and divided into four treatment groups: Control (treated with DMEM), EV71 $(\mathrm{MOI}=5), \operatorname{AST}(10 \mu \mathrm{g} / \mathrm{ml})$ and EV71 + ASTs (ASTs were added $2 \mathrm{~h}$ before EV71 infection). The cells were cultured for $24 \mathrm{~h}$ in an incubator at $37^{\circ} \mathrm{C}$ with $5 \% \mathrm{CO}_{2}$. The morphology of cells was observed and photographed using a light inverted microscope (magnification, x100; Nikon Corporation).

$C C K-8$ assay. Cells in the logarithmic growth phase were selected for the experiment. GES-1 cells were treated as aforementioned, then co-cultured with CCK-8 reagent for $2 \mathrm{~h}$. Absorbance was determined using a microplate reader (BioTek Instruments, Inc.) at $450 \mathrm{~nm}$ to calculate cell viability as follows: Cell viability $(\%)=\left(\right.$ Absorbance $_{\text {treatment }}{ }^{-}$ Absorbance $\left._{\text {control }}\right) /\left(\right.$ Absorbance $_{\text {black }}-$ Absorbance $\left._{\text {control }}\right) \times 100$. The blank well only contained medium and CCK- 8 reagent and the experiment was repeated three times.

Measurement of $\mathrm{LDH}$ release. $\mathrm{LDH}$ release was used to assess cell damage. GES-1 cells supernatant was collected using centrifugation (Eppendorf tubes; Eppendorf) at $400 \mathrm{x} \mathrm{g}$ for $5 \mathrm{~min}$ at room temperature. LDH was measured using an LDH detection kit, according to the manufacturer's instructions. LDH activity (\%) was calculated as follows: $\left(\mathrm{LDH}_{\text {treatment }}-\mathrm{LDH}_{\text {control }}\right) /\left(\mathrm{LDH}_{\max }-\mathrm{LDH}_{\text {control }}\right) \times 100$.

DAPI nuclear staining. After GES-1 cells were treated for $24 \mathrm{~h}$ as aforementioned, the culture medium was discarded. Cells were rinsed three times with PBS for 3 min each and then fixed with $4 \%$ paraformaldehyde for $20 \mathrm{~min}$ at $4^{\circ} \mathrm{C}$. After discarding paraformaldehyde, the cells were rinsed with PBS five times and then stained with DAPI $(1: 10,000)$ at room temperature for $5 \mathrm{~min}$. The cells were observed using a fluorescence microscope (magnification, x200).

Western blotting. Cells from the control and experimental groups were placed in 1.5-ml centrifuge tubes, then lysed with $60 \mu$ l RIPA lysis buffer (Beyotime Institute of Biotechnology) and the total protein concentration was determined using a 
BCA kit. Subsequently, $\sim 30 \mu \mathrm{g}$ total extracted protein (per well) from each group was separated using 12\% SDS-PAGE and then transferred to a nitrocellulose (NC) membrane. The $\mathrm{NC}$ membrane was blocked with 5\% skimmed milk for $2 \mathrm{~h}$ at room temperature and incubated with poly (ADP-ribose) polymerase (PARP; 1:1,000), c-PARP $(1: 1,000)$, caspase-3 (1:1,000), c-caspase-3 $(1: 1,000)$, NLRP3 $(1: 1,000)$, GSDMD $(1: 1,000)$, pro-caspase-1 $(1: 1,000)$, c-caspase-1 $(1: 1,000)$, pro-IL-1 $\beta$ $(1: 1,000)$ and virus structural protein VP1 $(1: 2,000)$ antibodies overnight at $4^{\circ} \mathrm{C}$. Following primary antibody incubation, the membrane was incubated with goat anti-rabbit IgG secondary antibody $(1: 5,000)$. The target proteins were observed using an ECL chromogenic kit by ChemiDoc ${ }^{\mathrm{TM}}$ MP Imaging System (Bio-Rad Laboratories, Inc.). ImageJ software (version 6.0; National Institutes of Health) was used for densitometry.

Reverse transcription-quantitative (RT-q) PCR. Total RNA was extracted from cells using TRIzol reagent (Invitrogen; Thermo Fisher Scientific, Inc.) and reverse-transcribed into cDNA using a BeyoRT II First Strand cDNA Synthesis kit (Beyotime Institute of Biotechnology) at $42^{\circ} \mathrm{C}$ for $60 \mathrm{~min}$, then left to stand at $80^{\circ} \mathrm{C}$ for $10 \mathrm{~min}$ to terminate the reaction. qPCR was performed using Hieff Universal Blue qPCR SYBR Green Master mix. The amplification reaction mixture consisted of $10 \mu 1$ 2X SYBR Green mix, a specific primer set (0.4 $\mu 1$ each), cDNA $(1 \mu \mathrm{g})$ and diethylpyrocarbonate (DEPC)-treated water to make the total volume of $20 \mu \mathrm{l}$. The following thermocycling conditions were used: Initial denaturation at $95^{\circ} \mathrm{C}$ for $2 \mathrm{~min}$; followed by 40 cycles of denaturation at $95^{\circ} \mathrm{C}$ for $10 \mathrm{sec}$ and extension at $55^{\circ} \mathrm{C}$ for $30 \mathrm{sec}$. GAPDH was used as an internal control and mRNA expression levels were analyzed using the $2^{-\Delta \Delta \mathrm{Cq}}$ method (27). The following primer pairs were used: EV71 forward, 5'-CGCACAGGGTCACTCAGAAC-3' and reverse, 5'-GCCCATTGCCACCAGTAGAC-3' and GAPDH forward, 5'-ACAACTTTGGCATTGTGGAA-3' and reverse, 5'-GATGCAGGGATGATGTTCTG-3'.

Statistical analysis. All experiments were independently repeated three times and data are presented as the mean \pm SD. One-way ANOVA followed by Tukey's post hoc was used to compare multiple groups, and Welch's test followed by Games-Howell post hoc test was applied to data with unequal variances; unpaired Student's t-test was used to compare statistical differences between two groups using SPSS 20.0 (SPSS, Inc.). $\mathrm{P}<0.05$ was considered to indicate a statistically significant difference.

\section{Results}

EV71 infection causes direct damage to cells. To assess whether EV71 infection damages GES-1 cells, GES-1 cells were infected with EV71 at various MOIs (0, 1, 3 and 5) for $24 \mathrm{~h}$. Cell morphology was then observed under an inverted microscope. The results showed that the cells became round, the cytoplasm shrank and the overall number of cells decreased (Fig. 1A). Cell viability was detected using a CCK-8 assay; EV71 infection caused GES-1 cell death in a dose-dependent manner $(\mathrm{P}<0.01$; Fig. 1B). LDH release was used to detect the damage to GES-1 cells; the results revealed that GES-1 cells were markedly damaged with the increase of MOIs, with MOI of 5 causing a significant increase in $\mathrm{LDH}$ release $(\mathrm{P}<0.01$; Fig. 1C). It was concluded that EV71 infection caused direct damage to GES-1 cells.

EV71 induces apoptosis and pyroptosis. Considering the association between EV71 and induction of PCD $(8,28)$, EV71-induced cell apoptosis and pyroptosis were investigated. GES-1 cells were infected with EV71 at different MOIs for $24 \mathrm{~h}$. The expression of apoptosis-associated proteins PARP, c-PARP, Caspase-3 and c-caspase-3 were analyzed using western blotting. Levels of PARP and caspase-3 protein decreased following EV71 infection ( $\mathrm{P}<0.01$; Fig. $2 \mathrm{~A})$. The pyroptosis-associated proteins pro-GSDMD and pro-IL-1 $\beta$ were also detected using western blotting. The results showed that pro-GSDMD and pro-IL-1 $\beta$ in GES- 1 cells were decreased following EV71 infection ( $\mathrm{P}<0.01$; Fig. $2 \mathrm{~B})$. It was concluded that EV71 induces apoptosis and pyroptosis in GES-1 cells.

ASTs alleviate EV71 infection-induced cell damage. To assess the protective effect of ASTs on EV71 infection-induced cell damage, GES-1 cells were treated with ASTs $(10 \mu \mathrm{g} / \mathrm{ml})$ $2 \mathrm{~h}$ before infection with EV71 (MOI=5, as $>50 \%$ of GES-1 cells were damaged) for $24 \mathrm{~h}$. GES-1 cell morphology was observed under a microscope. The number of ASTs-treated cells was higher than that of cells infected with EV71 (Fig. 3A). The viability of AST-treated cells infected with EV71 was detected using CCK-8 assay; the results showed that ASTs improved the viability of EV71-infected GES-1 cells $(\mathrm{P}<0.01$; Fig. 3B). EV71 infection-induced damage in AST-treated cells was measured using a LDH assay. The results showed that ASTs alleviated EV71 infection-induced cell damage $(\mathrm{P}<0.01$; Fig. $3 \mathrm{C})$.

ASTs inhibit GES-1 cell apoptosis. To assess whether ASTs inhibit GES-1 cell apoptosis, DAPI was used to stain the nucleus of GES-1 cells. The number of cells was found to be decreased and the nucleus shrank following EV71 infection. However, these pathological changes in the nucleus were reversed following treatment with ASTs (Fig. 4A). In addition, changes in the expression levels of apoptotic proteins were measured using western blotting. The results revealed lower c-PARP and c-caspase-3 levels in EV71-infected and AST-treated GES-1 cells compared with the control group $(\mathrm{P}<0.01$; Fig. 4B and $\mathrm{C})$.

ASTs inhibit pyroptosis in GES-1 cells. To determine whether ASTs inhibit GES-1 cell pyroptosis, western blotting was used to detect marker proteins of pyroptosis. Compared with the control group, the expression levels of pyroptosis-associated proteins NLRP3 and c-caspase-1 were lower, and those of pro-GSDMD, pro-caspase-1 and pro-IL-1 $\beta$ was significantly higher in EV71-infected and AST-treated GES-1 cells $(\mathrm{P}<0.05$ or $\mathrm{P}<0.01$; Fig. $5 \mathrm{~A}$ and $\mathrm{B}$ ).

ASTs inhibit EV71 replication. ASTs inhibited apoptosis and pyroptosis to alleviate EV71-induced cell damage. Therefore, it was investigated whether the effect of ASTs on cell pyroptosis and apoptosis was mediated by regulation of EV71. The expression of the EV71 structural protein, VP1, was detected using western blotting and VP1 mRNA expression levels were 
A
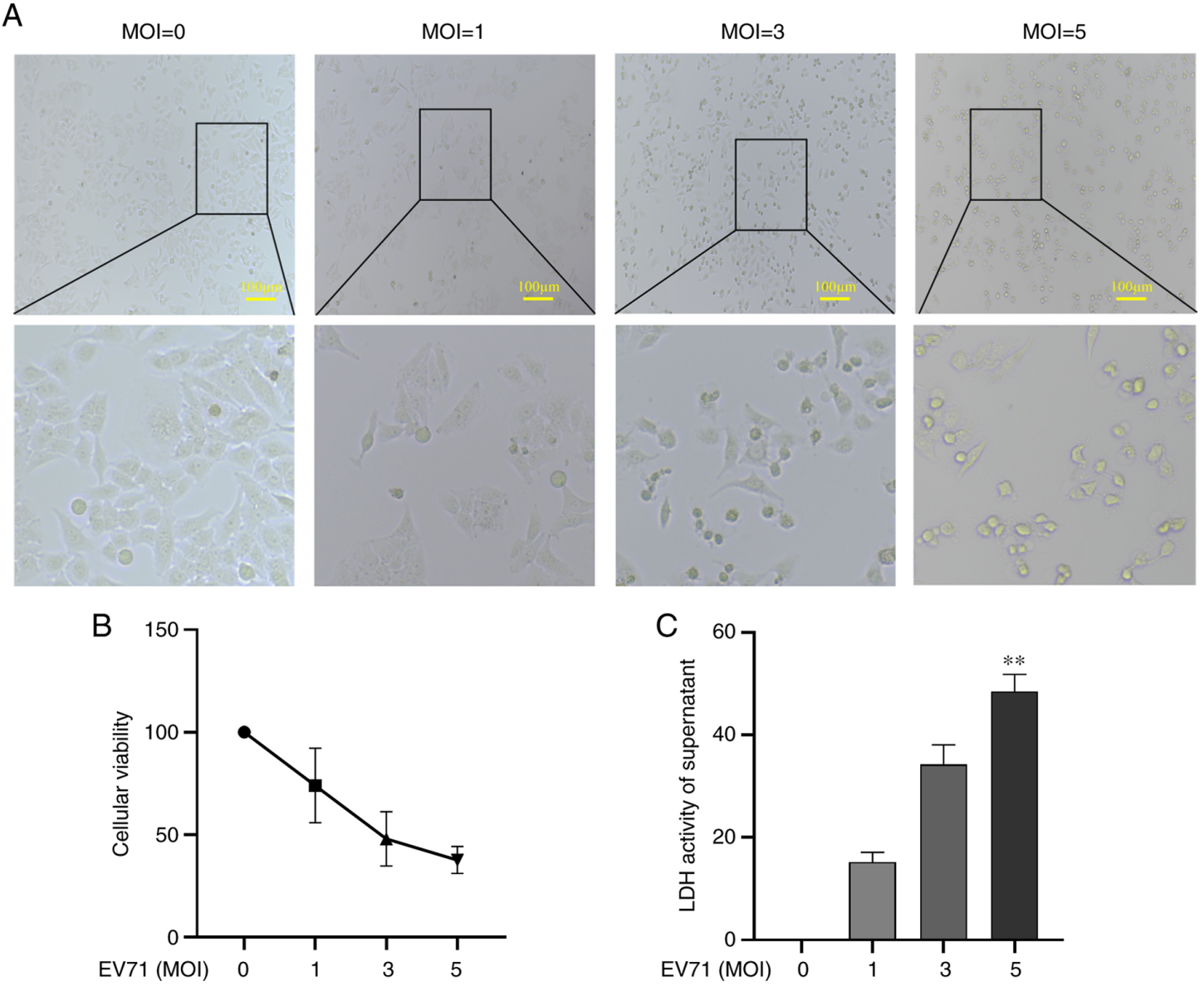

Figure 1. EV71 infection causes direct damage to cells. (A) GES-1 cells were infected with EV71 at MOIs of 0,1,3 and 5. Cell morphology was observed under an inverted microscope (magnification, x100). (B) GES-1 cell activity was evaluated using a Cell Counting Kit- 8 assay. (C) GES-1 cell damage was evaluated by $\mathrm{LDH}$ release. Data are presented as the mean $\pm \mathrm{SD}(\mathrm{n}=3) .{ }^{* *} \mathrm{P}<0.01$ vs. 0 . EV71, enterovirus 71 ; LDH, lactate dehydrogenase; MOI, multiplicity of infection.

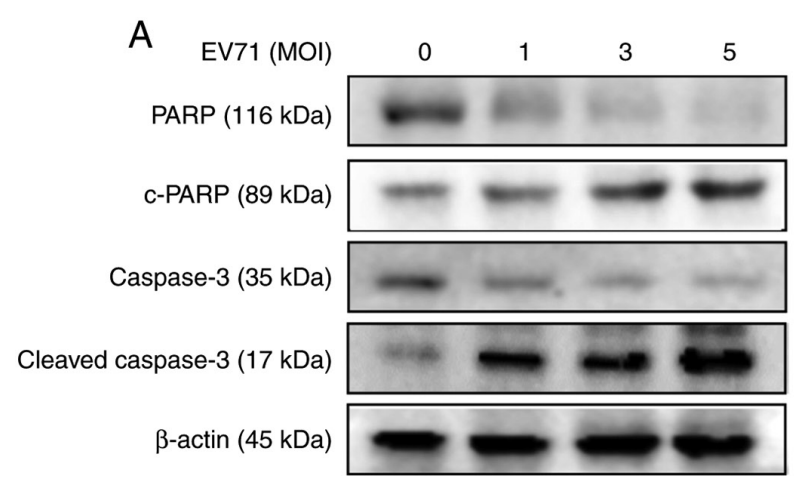

B
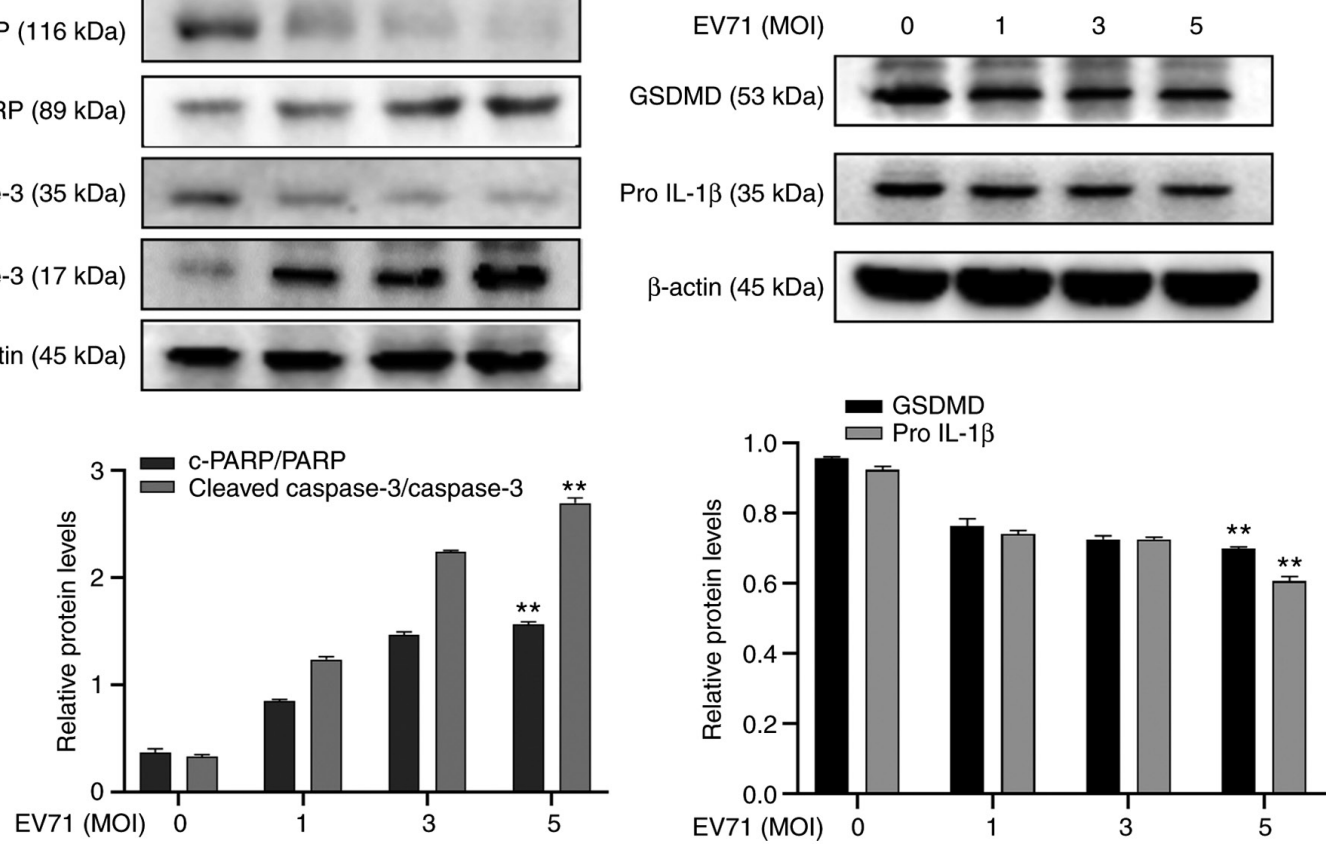

Figure 2. EV71 induces apoptosis and pyroptosis. GES-1 cells were harvested and total protein was extracted to determine the expression of (A) apoptosis-associated proteins PARP, c-PARP, Caspase-3 and c-caspase-3 and (B) pyroptosis-associated proteins GSDMD and pro-IL-1 $\beta$ using western blotting. The expression of $\beta$-actin served as an internal control. Data are presented as the mean \pm SD $(n=3)$. ${ }^{* *} \mathrm{P}<0.01$ vs. 0 . EV71, enterovirus 71 ; PARP, poly (ADP-Ribose) polymerase; GSDMD, gasdermin D protein; MOI, multiplicity of infection; c-, cleaved. 
A

Control
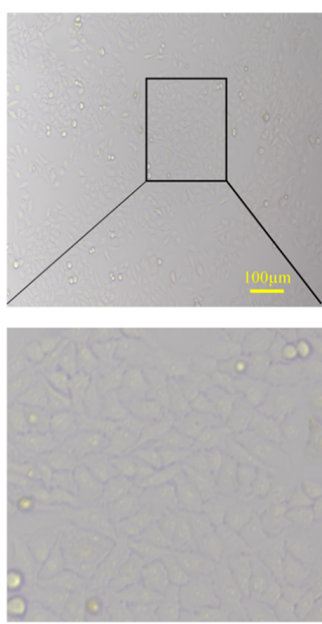

B

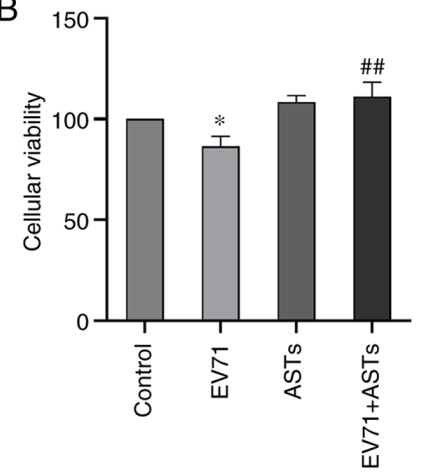

ASTs
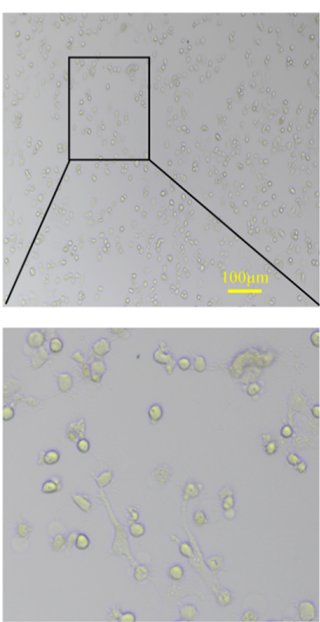

C
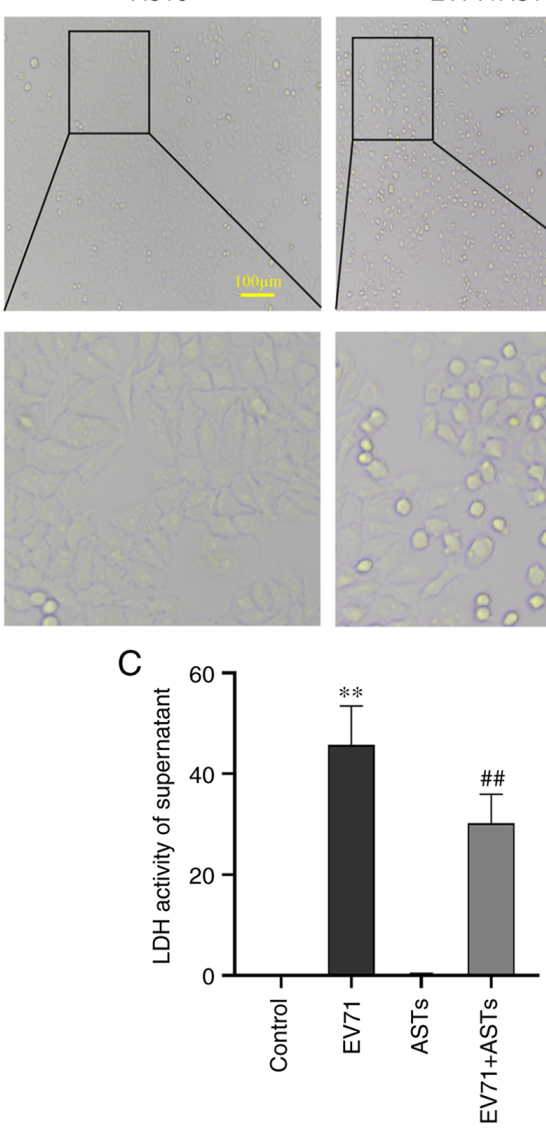

Figure 3. ASTs alleviate EV71 infection-induced cell damage. (A) To determine the protective effect of ASTs, GES-1 cells were treated with ASTs prior to EV71 infection for $2 \mathrm{~h}$. Cell morphology was observed using a light microscope (magnification, x100). (B) Cell Counting Kit- 8 assay was performed to detect the viability of treated cells. (C) LDH release was measured to assess cell damage. Data are presented as the mean $\pm \mathrm{SD}(\mathrm{n}=3)$. ${ }^{*} \mathrm{P}<0.05$ and ${ }^{* *} \mathrm{P}<0.01$ vs. control; ${ }^{\# \#} \mathrm{P}<0.01$ vs. EV71. ASTs, total astragalosides; EV71, enterovirus 71; LDH, lactate dehydrogenase.

A

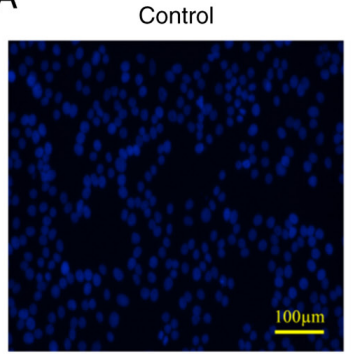

EV71

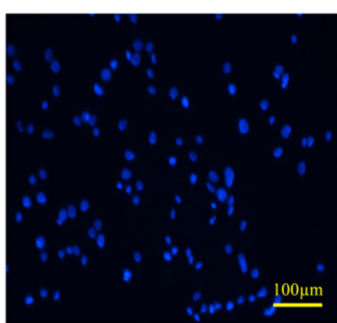

ASTs

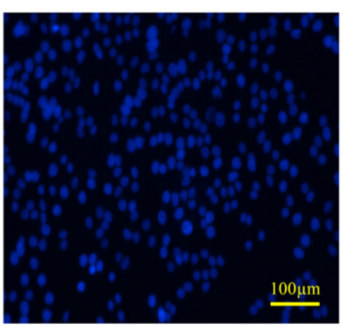

EV71+ASTs
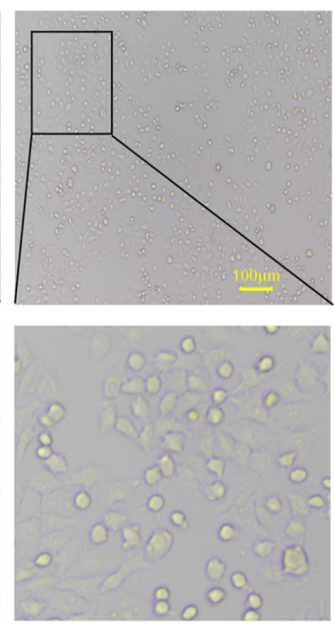

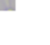 \\ .}


A

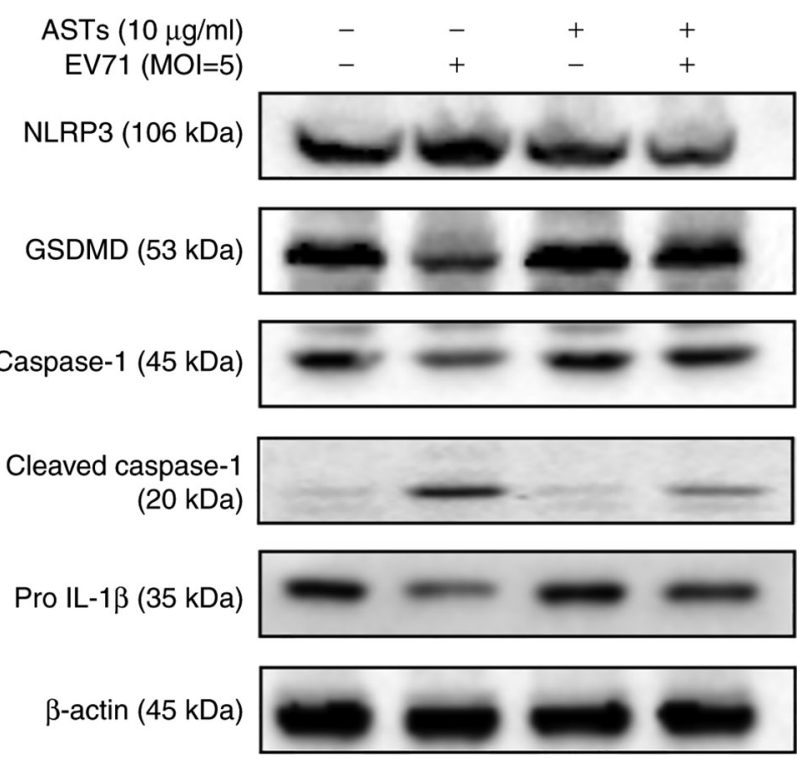

B

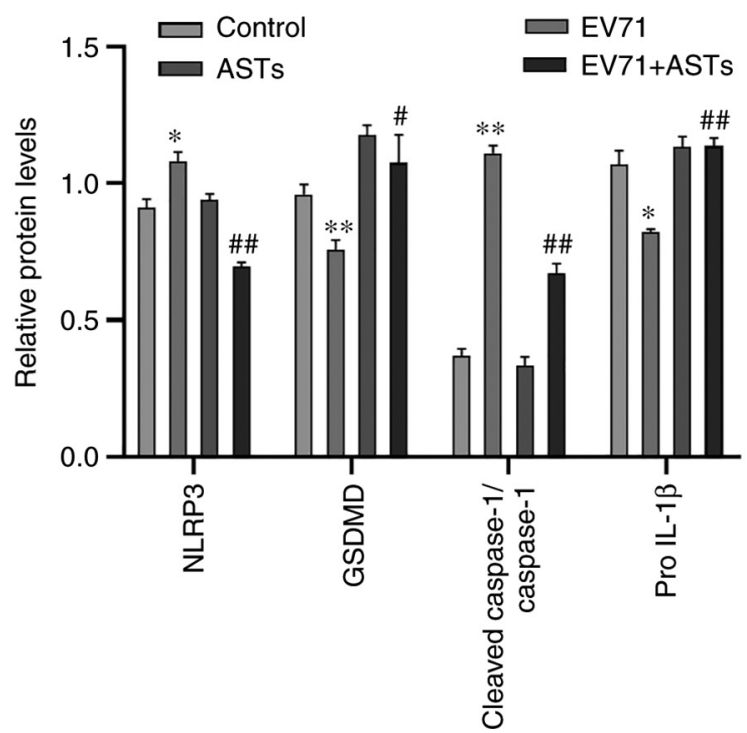

Figure 5. ASTs inhibit pyroptosis in GES-1 cells. (A) To determine the effect of ASTs on pyroptosis, GES-1 cells were seeded in culture plates and infected with EV71 at a MOI of 5 in AST medium. The cells were collected and total protein was measured using western blotting. Anti-NLRP3, anti-GSDMD, anti-pro-caspase-1, anti-c-caspase-1 and anti-IL-1 $\beta$ antibodies were used to analyze the levels of pyroptosis in cells. (B) Relative protein expression levels are also presented. Data are presented as the mean $\pm \mathrm{SD}(\mathrm{n}=3)$. ${ }^{*} \mathrm{P}<0.05$ and ${ }^{* *} \mathrm{P}<0.01$ vs. control; ${ }^{\#} \mathrm{P}<0.05$ and ${ }^{\# \#} \mathrm{P}<0.01$ vs. EV71. ASTs, total astragalosides; EV71, enterovirus 71; NLRP3, NLR family, pyrin domain containing 3; GSDMD, gasdermin D protein; MOI, multiplicity of infection; c-, cleaved.

measured using RT-qPCR. Results showed that the protein and mRNA expression of VP1 was decreased following AST treatment $(\mathrm{P}<0.01$ or $\mathrm{P}<0.05$, respectively; Fig. $6 \mathrm{~A}$ and $\mathrm{B})$. A TCID50 assay was used to detect EV71 release in the cell culture supernatant. The results showed that ASTs inhibited EV71 release in GES-1 cells ( $\mathrm{P}<0.01$; Fig. 6C). These results showed that ASTs inhibited EV71 replication and release.

\section{Discussion}

HFMD is a pediatric disease caused by EV71 infection that is prevalent worldwide (29). The incidence of HFMD in China has been rising since 2008, but there are no specific antiviral drugs for the control of EV71 infection (30). Therefore, identifying effective antiviral agents against EV71 viral infection is urgent.

Previous studies have shown that the active components of certain plants exert antiviral effects. For example, Kalanchoe gracilis leaf extract inhibits both Cox A16 and EV71 infection in vivo and in vitro by inhibiting viral non-structural protein $2 \mathrm{~A}$ protease activity (31). Apigenin directly binds to heterogeneous nuclear ribonucleoprotein A2 protein to prevent EV71 infection (32). Oblongifolin $M$ extracted from Garcinia oblongifolia suppresses EV71 replication by downregulating endoplasmic reticulum resident protein 57 (33). Baicalin inhibits replication of EV71 by inhibiting expression of EV1 3D polymerase (34). As an active component of AM, ASTs exert obvious protective effects in cell injury. Li et al (24) found that ASTs protect the kidney from shock wave-induced oxidation injury. In a previous study, the EV71 receptors scavenger receptor class B member 2 and P-selectin glycoprotein ligand-1 were found to be distributed in the human gastrointestinal tract, and the stomach was the primary
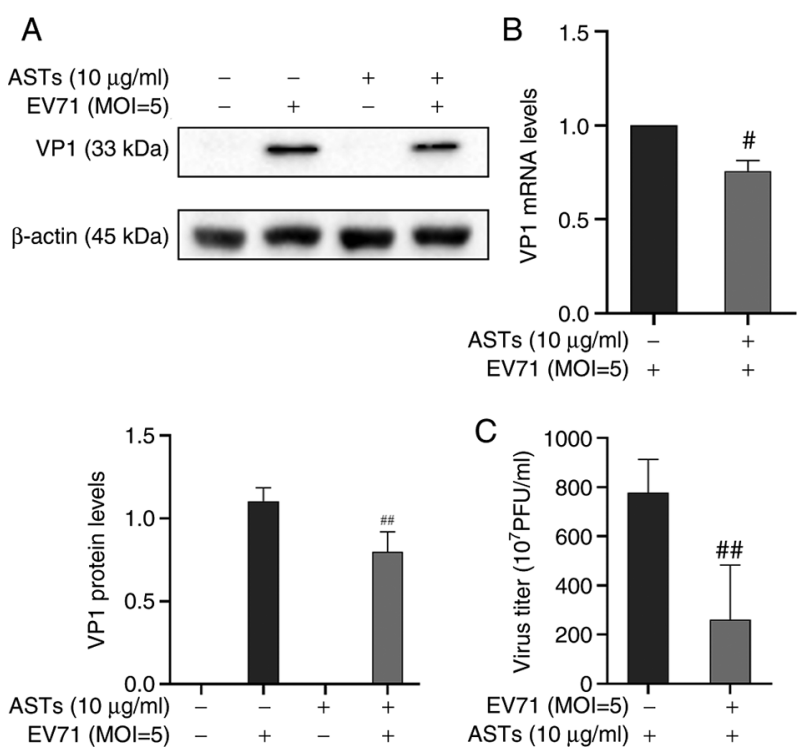

Figure 6. ASTs inhibit EV71 replication. (A) To determine whether ASTs inhibit replication of EV71, GES-1 cells were incubated with EV71 and ASTs. Western blotting was used to assess the expression levels of VP1 and host $\beta$-actin. (B) Reverse transcription-quantitative PCR was used to detect levels of VP1 mRNA. (C) Supernatant from treated cells was collected, and a median tissue culture infectious dose assay was performed to detect the virus titer. Data are presented as the mean $\pm \mathrm{SD}(\mathrm{n}=3)$. ${ }^{\#} \mathrm{P}<0.05$ and ${ }^{\# \#} \mathrm{P}<0.01$ vs EV71. ASTs, total astragalosides; EV71, enterovirus 71; VP1, virus structural protein; MOI, multiplicity of infection.

site of EV71 infection (35). Our previous experiment showed that GES-1 cells are sensitive to EV71 (36). However, the present study discovered that EV71 infection led to shrinkage of GES-1 cell cytoplasm and decrease in cell number, while ASTs alleviated GES-1 cell damage. 
Apoptosis is an autonomous type of PCD (9). EV71, hepatitis $\mathrm{B}$ and $\mathrm{C}$ and other viruses induce apoptosis $(7,37,38)$. Viruses tend to use host cells to induce apoptosis of tissue or immune cells to infect the body (39). The present study found that EV71 infection decreased expression levels of apoptosis marker proteins caspase- 3 and PARP in GES-1 cells, and ASTs ameliorated this effect. Thus, the present study showed that EV71 induced apoptosis of GES-1 cells and ASTs suppressed this process. These results suggested that ASTs inhibited EV71 replication and virus-induced cytopathic effects, such as apoptosis and pyroptosis. However, the exact mechanism requires further in vivo studies.

A variety of cytokines, such as TNF- $\alpha$, IL-1, IL-6, IL-12, IFN- $\alpha$, IFN- $\beta$, monocyte chemoattractant protein- 1 and IL- 8 are rapidly produced in the body following infection with microorganisms, which is a significant cause of acute respiratory distress syndrome and multiple organ failure (40). EV71 infection induces IL-4,IL-6, IL-12, TNF and IFN production and pyroptosis $(36,41)$. Cells undergoing pyroptosis have been shown to produce IL-1 $\beta$ and IL-18 cytokines, which induce inflammation in the body (42). The present study revealed that EV71 infection induced an increase in the protein level of NLRP3, while it decreased the protein expression levels of GSDMD, pro-caspase-1 and pro-IL-1 $\beta$ in GES-1 cells. Conversely, ASTs inhibited pyroptosis and IL-1 $\beta$ production to suppress EV71-induced inflammation. Thus, ASTs exerted both antiviral and anti-inflammatory effects, which may support the further clinical application of ASTs in the treatment of EV71-induced HFMD. Future studies should aim to provide insight into the mechanism of AST-induced antiviral effects in vitro, as well as searching for potential antiviral targets. The present results will be further demonstrated in animal models. However, an animal model of virus infection requires a special biosafety laboratory and there are few laboratories in the world with a license. The present cell experiments were performed in a Biosafety Level II laboratory (43).

In conclusion, the findings of the present study suggested that EV71 infection may induce GES-1 cell apoptosis and pyroptosis, while ASTs may suppress these processes and alleviate cytotoxicity. This protective effect may be due to the ability of ASTs to suppress the replication and release of EV71 in GES-1 cells.

\section{Acknowledgements}

Not applicable.

\section{Funding}

The present study was supported by the National Natural Science Foundation of China (grant no. 81301426), the Provincial Natural Science Foundation of Shanxi (grant no. 201901D111329), the Mega Research and Development Projects of Lüliang (grant no. 2020SHFZ38), The Program of Fenyang College, Shanxi Medical University (grant no. 2020B01) and The Key Laboratory Platform Construction Projects of Lüliang (grant no. 2020ZDSYS17).

\section{Availability of data and materials}

The datasets used and/or analyzed during the current study are available from the corresponding author on reasonable request.

\section{Authors' contributions}

QL, XZ and JH conceived and designed the experiments. XZ, $\mathrm{JH}, \mathrm{CS}, \mathrm{JD}$ and $\mathrm{QH}$ performed the experiments. JH analyzed the data. XZ and JH wrote the manuscript. QL and XZ revised the manuscript. All authors have read and approved the final manuscript. XZ and JH confirm the authenticity of all the raw data.

\section{Ethics approval and consent to participate}

Not applicable.

\section{Patient consent for publication}

Not applicable.

\section{Competing interests}

The authors declare that they have no competing interests.

\section{References}

1. Gao W, Hou M, Liu X, Li Z, Yang Y and Zhang W: Induction of SOCS expression by EV71 infection promotes EV71 replication. Biomed Res Int 2020: 2430640, 2020.

2. Fu M, Bai J, Gao S, Chang Z, Zhou X and Long JE: Construction and characterization of an infectious cDNA clone of enterovirus 71: A rapid method for rescuing infectious virus based on stable cells expressing T7 polymerase. Arch Virol 166: 627-632, 2021.

3. Hu Y, Xu Y, Huang Z, Deng Z, Fan J, Yang R, Ma H, Song J and Zhang Y: Transcriptome sequencing analysis of SH-SY5Y cells infected with EV71 reveals the potential neuropathic mechanisms. Virus Res 282: 197945, 2020.

4. Fu Y, Zhang L, Zhang F, Tang T, Zhou Q, Feng C, Jin Y and Wu Z: Exosome-mediated miR-146a transfer suppresses type I interferon response and facilitates EV71 infection. PLoS Pathog 13: e1006611, 2017.

5. Ampomah PB and Lim LHK: Influenza A virus-induced apoptosis and virus propagation. Apoptosis 25: 1-11, 2020.

6. Xie WH, Ding J, Xie XX, Yang XH, Wu XF, Chen ZX, Guo QL, Gao WY, Wang XZ and Li D: Hepatitis B virus X protein promotes liver cell pyroptosis under oxidative stress through NLRP3 inflammasome activation. Inflamm Res 69: 683-696, 2020.

7. Hu X, Jiang J, Ni C, Xu Q, Ye S, Wu J, Ge F, Han Y, Mo Y, Huang D and Yang L: HBV integration-mediated cell apoptosis in HepG2.2.15. J Cancer 10: 4142-4150, 2019.

8. Wang Y, Qin Y, Wang T, Chen Y, Lang X, Zheng J, Gao S, Chen S, Zhong X, Mu Y, et al: Pyroptosis induced by enterovirus 71 and coxsackievirus B3 infection affects viral replication and host response. Sci Rep 8: 2887, 2018.

9. Ding Q, Zhang W, Cheng C, Mo F, Chen L, Peng G, Cai X, Wang J, Yang S and Liu X: Dioscin inhibits the growth of human osteosarcoma by inducing G2/M-phase arrest, apoptosis, and GSDME-dependent cell death in vitro and in vivo. J Cell Physiol 235: 2911-2924, 2020.

10. Karam JA, Lotan Y, Karakiewicz PI, Ashfaq R, Sagalowsky AI, Roehrborn CG and Shariat SF: Use of combined apoptosis biomarkers for prediction of bladder cancer recurrence and mortality after radical cystectomy. Lancet Oncol 8: 128-136, 2007.

11. Häcker G: The morphology of apoptosis. Cell Tissue Res 301: 5-17, 2000

12. Wyllie AH, Kerr JF and Currie AR: Cell death: The significance of apoptosis. Int Rev Cytol 68: 251-306, 1980.

13. Song F, Yu X, Zhong T, Wang Z, Meng X, Li Z, Zhang S, Huo W, Liu X, Zhang Y, et al: Caspase-3 inhibition attenuates the cytopathic effects of EV71 infection. Front Microbiol 9: 817, 2018.

14. Cookson BT and Brennan MA: Pro-inflammatory programmed cell death. Trends Microbiol 9: 113-114, 2001. 
15. Brennan MA and Cookson BT: Salmonella induces macrophage death by caspase-1-dependent necrosis. Mol Microbiol 38: 31-40, 2000.

16. Gaul S, Leszczynska A, Alegre F, Kaufmann B, Johnson CD, Adams LA, Wree A, Damm G, Seehofer D, Calvente CJ, et al: Hepatocyte pyroptosis and release of inflammasome particles induce stellate cell activation and liver fibrosis. J Hepatol 74: 156-167, 2021.

17. Duan G, Yang H, Shi L, Sun W, Sui M, Zhang R, Wang X, Wang F, Zhang W, Xi Y and Fan Q: Serum inflammatory cytokine levels correlate with hand-foot-mouth disease severity: A nested serial case-control study. PLoS One 9: e112676, 2014.

18. Han J, Wang Y, Gan X, Song J, Sun P and Dong XP: Serum cytokine profiles of children with human enterovirus 71-associated hand, foot, and mouth disease. J Med Virol 86: 1377-1385, 2014.

19. James DS: The multisystem adverse effects of NSAID therapy. J Am Osteopath Assoc 99 (Suppl 11): S1-S7, 1999.

20. Polderman JA, Farhang-Razi V, Van Dieren S, Kranke P, DeVries JH, Hollmann MW, Preckel B and Hermanides J: Adverse side effects of dexamethasone in surgical patients. Cochrane Database Syst Rev 8: CD011940, 2018.

21. Zhang X, Liang T, Yang W, Zhang L, Wu S, Yan C and Li Q Astragalus membranaceus injection suppresses production of interleukin- 6 by activating autophagy through the AMPK-mTOR pathway in lipopolysaccharide-stimulated macrophages. Oxid Med Cell Longev 2020: 1364147, 2020.

22. Sai YP, Song YC, Chen XX, Luo X, Liu J and Cui WJ Protective effect of astragalosides from radix astragali on adriamycin-induced podocyte injury. Exp Ther Med 15: 4485-4490, 2018.

23. OuYang Y, Huang J, OuYang Z and Kang J: Enrichment and purification process of astragalosides and their anti-human gastric cancer MKN-74 cell proliferation effect. Afr Health Sci 14 22-27, 2014.

24. Li X, He D, Zhang L, Cheng X, Sheng B and Luo Y: A novel antioxidant agent, astragalosides, prevents shock wave-induced renal oxidative injury in rabbits. Urol Res 34: 277-282, 2006.

25. Li C, Yang F, Liu F, Li D and Yang T: NRF2/HO-1 activation via ERK pathway involved in the anti-neuroinflammatory effect of astragaloside IV in LPS induced microglial cells. Neurosci Lett 666: 104-110, 2018.

26. Cheng JH, Sun YJ, Zhang FQ, Zhang XR, Qiu XS, Yu LP, Wu YT and Ding C: Newcastle disease virus NP and P proteins induce autophagy via the endoplasmic reticulum stress-related unfolded protein response. Sci Rep 6: 24721, 2016

27. Li Q, Cheng F, Zhou K, Fang L, Wu J, Xia Q, Cen Y, Chen J and Qing Y: Increased sensitivity to TNF- $\alpha$ promotes keloid fibroblast hyperproliferation by activating the NF- $\kappa \mathrm{B}$, JNK and p38 MAPK pathways. Exp Ther Med 21: 502, 2021.

28. Xu T, Li Y, Wu HL, Chen H, Wu H, Guo M, Zhao M, Wang C, Lin T, Lin Z, et al: The inhibition of enterovirus 71 induced apoptosis by durvillaea antarctica through P53 and STAT1 signaling pathway. J Med Virol 93: 3532-3538, 2021.

29. You L, Chen J, Liu W, Xiang Q, Luo Z, Wang W, Xu W, Wu K, Zhang Q, Liu Y and Wu J: Enterovirus 71 induces neural cell apoptosis and autophagy through promoting ACOX1 downregulation and ROS generation. Virulence 11: 537-553, 2020.
30. Ma JQ, Sun YZ, Ming QL, Tian ZK, Yang HX and Liu CM: Ampelopsin attenuates carbon tetrachloride-induced mouse liver fibrosis and hepatic stellate cell activation associated with the SIRT1/TGF- $\beta 1 /$ Smad3 and autophagy pathway. Int Immunopharmacol 77: 105984, 2019.

31. Wang CY, Huang SC, Zhang Y, Lai ZR, Kung SH, Chang YS and Lin CW: Antiviral ability of Kalanchoe gracilis leaf extract against enterovirus 71 and coxsackievirus A16. Evid Based Complement Alternat Med 2012: 503165, 2012.

32. Zhang W, Qiao H, Lv Y, Wang J, Chen X, Hou Y, Tan R and Li E: Apigenin inhibits enterovirus-71 infection by disrupting viral RNA association with trans-acting factors. PLoS One 9: e110429, 2014.

33. Wang M, Dong Q, Wang H, He Y, Chen Y, Zhang H, Wu R, Chen X, Zhou B, He J, et al: Oblongifolin M, an active compound isolated from a Chinese medical herb Garcinia oblongifolia, potently inhibits Enterovirus 71 reproduction through downregulation of ERp57. Oncotarget 7: 8797-8808, 2016.

34. Li X, Liu Y, Wu T, Jin Y, Cheng J, Wan C, Qian W, Xing F and Shi W: The antiviral effect of baicalin on enterovirus 71 in vitro. Viruses 7: 4756-4771, 2015.

35. Jiao XY, Guo L, Huang DY, Chang XL and Qiu QC: Distribution of EV71 receptors SCARB2 and PSGL-1 in human tissues. Virus Res 190: 40-52, 2014.

36. Cao L, Zhang X, Yuan S, Cheng K and Zhang X: Autophagy induced by enterovirus 71 regulates the production of IL-6 through the p38MAPK and ERK signaling pathways. Microb Pathog 131: 120-127, 2019.

37. Li H, Bai Z, Li C, Sheng C and Zhao X: EV71 infection induces cell apoptosis through ROS generation and SIRT1 activation. J Cell Biochem 121: 4321-4331, 2020.

38. Guo X, Liu WL, Yang D, Shen ZQ, Qiu ZG, Jin M and Li JW: Hepatitis $C$ virus infection induces endoplasmic reticulum stress and apoptosis in human fetal liver stem cells. J Pathol 248: $155-163,2019$.

39. Iannello A, Debbeche O, Martin E, Attalah LH, Samarani S and Ahmad A: Viral strategies for evading antiviral cellular immune responses of the host. J Leukoc Biol 79: 16-35, 2006.

40. Guo XJ and Thomas PG: New fronts emerge in the influenza cytokine storm. Semin Immunopathol 39: 541-550, 2017.

41. Shao $\mathrm{P}, \mathrm{Wu} X, \mathrm{Li} \mathrm{H}, \mathrm{Wu} \mathrm{Z}$, Yang $\mathrm{Z}$ and Yao H: Clinical significance of inflammatory cytokine and chemokine expression in hand, foot and mouth disease. Mol Med Rep 15: 2859-2866, 2017.

42. Zhuo L, Chen X, Sun Y, Wang Y, Shi Y, Bu L, Xia W, Han J, Chen D and Li X: Rapamycin inhibited pyroptosis and reduced the release of IL-1 $\beta$ and IL-18 in the septic response. Biomed Res Int 2020: 5960375, 2020.

43. Kruse RH, Puckett WH and Richardson JH: Biological safety cabinetry. Clin Microbiol Rev 4: 207-241, 1991.

This work is licensed under a Creative Commons Attribution-NonCommercial-NoDerivatives 4.0 International (CC BY-NC-ND 4.0) License. 